\title{
SEISMIC TORSIONAL EFFECTS ON BUILDINGS
}

\author{
D.G. Elms *
}

\begin{abstract}
SUMMARY
This paper gives the background to the seismic torsional provisions of the New Zealand Loadings Code. These provisions attempt to deal with three effects: accidental eccentricity, torsional ground motion, and coupling between torsional and translational modes. The first two effects lead to an effective eccentricity of one-tenth of the width of a building, while the third leads to a parabolic function of the calculated eccentricity: this was obtained by applying existing results for torsional amplification to a number of typical building structures.
\end{abstract}

\section{INTRODUCTION}

Horizontal torsional effects in buildings are not well understood. However, they are thought to have contributed to a number of serious failures. Torsion causes an increase in shear at the periphery of a building, and it also appears to have caused torsional failure in some columns. During dynamic testing, even completely symmetric buildings or models have usually shown considerable torsional effects at some stage.

Most codes seem reluctant to make detailed recommendations on torsion. There are, however, some exceptions: Mexico, Venezuela and Peru, for instance, give formulae to be used with a static design approach (1). The Uniform Building Code requires an allowance of $5 \%$ of the maximum overall plan dimension for accidental eccentricity. The SEAOC Code says 'Provisions shall be made for the increase in shear resulting from the horizontal torsion due to an eccentricity between the centre of mass and the centre of rigidity.' This has a minimum value of 5 o where diaphragms are used. Negative torsional shears are to be neglected. The 1973 revision requires that 'The distribution of the lateral forces in structures which have highly irregular shapes ... shall be determined considering the dynamic characteristics of the structure." The commentary indicates that this includes torsion, but goes on to say that as 'A three-dimensional analysis (is) ... generally not practical, joints should be provided to separate the various blocks of the structure.' An adequate static design approach is not given, and neither is mention made of the possibility of torsional column failure.

In drafting the present New Zealand Code, the matter of torsion was considered carefully, and it was decided that despite lack of precedent in most codes a static design formula should be provided for use, both directly in the design of suitable buildings, and also as an order-of-magnitude check for buildings designed using a

* Reader, Department of Civil Engineering, University of Canterbury. dynamic analysis.

The present code envisages three types of design approach: a fully static analysis for regular buildings, within certain limitations; a mixed approach in which transverse effects are computed using a two-dimensional modal analysis, using the static formula to give torsional effects; and a full three-dimensional modal analysis. For moderately unbalanced buildings, if a dynamic analysis is carried out the design torsional effects may not be taken as less than those produced by the static requirements.

The Commentary to the New Zealand Code gives broad limitations to the applicability of the static method alone, namely that it is intended to apply only to buildings which are reasonably regular both in plan and from floor to floor. No attempt has been made to define a 'reasonably regular building' with precision: it should be obvious if a building is a 'highly irregular structure', otherwise the engineer must use his own judgement. For a structure, even if regular, with more than, say, twelve storeys, the static approach cannot be used with confidence as it is based primarily on an analysis of single-storey behaviour. One specific limitation is given in the commentary: buildings with a structural type factor greater than or equal to 1.6, that is, nonductile buildings, are limited to a maximum eccentricity of $0.3 b$, where $b$ is the building width. In any case, although the applicability of a static approach is limited, dynamic methods will not necessarily give a wholly trustworthy result: torsional effects are so uncertain that even a full 3-dimensional computer analysis may not give a good prediction of the dynamic behaviour of a highly irregular building.

\section{STATIC APPROACH}

\subsection{Use of the Code Formulae}

For a static analysis, the code requires that at a given level in a building, two design eccentricities shall be given by the following formulae. 


$$
\begin{aligned}
& e_{d 1}=1.7 e_{s}-e_{s}{ }^{2} / b+0.1 b \\
& e_{d 2}=e_{s}-0.1 b
\end{aligned}
$$

whichever is the more unfavourable, where $e_{s}$ is the static eccentricity and $b$ is the breadth of the building.

The formulae in effect define two extreme positions at which the lateral force might act. The two alternative force positions are shown as $\mathrm{F}_{1}$ and $\mathrm{F}_{2}$ in $\mathrm{Fig}$. 1 . For $e_{s}<0.1 b F_{2}$ will of course be applied on the side of the centre of rigidity away from the centre of mass. Figs. $I b$ and $1 c$ show that $F_{1}$ gives the critical case for the left-hand side while $\mathrm{F}_{2}$ gives the worst case for the right. This will not always be so. In some instances $F_{I}$ will be critical for both ends of the building. Note that the old restriction of NZS 1900 Chapt. 8, that torsional effects may not be used to reduce shear at any point, is no longer in the code.

\subsection{Background to the Formulae}

The static provisions of the code stemmed originally from a suggestion by Newmark and Rosenblueth (2) that within certain limits, a static analysis could be based on the relations

$$
\begin{aligned}
e_{d} & =1.5 e_{s}+a b \\
\text { or } e_{d} & =e_{s}-\alpha b
\end{aligned}
$$

where $\alpha$ is the order 0.05 to 0.10 . The main disadvantages of these formulae are that $1.5 \mathrm{e}_{\mathrm{s}}$ is severe for large values of $e_{s}$ (though it has been argued by some that this is justifiable as a penalty against highly assymetric buildings), and that the value of $\alpha$ is subject to some doubt. The Acapulco, Mexico, code and the Venezuelan code both use $e_{d}=1.5 e s \pm 0.05 b$, while Peru uses $e_{\bar{d}}=1.5 e_{s}+8.05 b$ or $e_{d}=e_{s}$ $0.05 \mathrm{~b}$ but with no shear increase allowed

The code provisions have to cover two sources of torsional effects: accidental eccentricity due to uncertainty as to both excitation and response; and coupling of torsional and translational modes where the natural periods are close.

\subsection{Accidental Eccentricity}

The term $0.1 \mathrm{~b}$ in equations ( 1 ) and (2) has contributions coming from a number of sources. These are considered individually as follows :

(a) Accidental variations in centre of rigidity - The calculated position of the centre of rigidity may differ from the actual for a number of reasons, such as the modelling assumptions made, limitations of stiffness calculations, effects of secondary structure, variations in dimensions and material properties, foundation effects and, for severe earthquakes, asymmetric failure of torsion-resisting elements. greatest of these effects. Taking a peripheral frame building $40 \times 20 \mathrm{~m}$ with 18 peripheral columns, the complete loss of stiffness of two of the end columns shifts the centre of rigidity by about $0.05 \mathrm{~b}$. In general, this would be an increase in eccentricity as the centre of rigidity will shift away from the damaged area. In a sense, this is an unstable situation, leading to more strain in the already distressed region. For this reason Newmark (3) advocates particular care being taken in the design of corner columns and peripheral shear walls. To take care of this effect. an eccentricity of $0.04 \mathrm{~b}$ is assumed.

The other accidental stiffness effects are more difficult to estimate. In probabilistic terms, the expected value of the accidental shift of centre of rigidity can be taken as zero: that is, there is an equal likelihood of it moving either way. However, the effects will contribute to the total variance of the eccentricity and hence will increase its design value. Let us estimate the increase to be $0.02 \mathrm{~b}$. In making this estimate an average rather than a conservative value is sought: an adequate safety factor has already been achieved in calculating the base shear coefficient $c d$.

(b) Accidental variations in centre of mass - This effect is likely to be small as the centre of mass can be found more reliably than the centre of stiffness. However, this may not be true for warehouses, storage buildings and other structures with a large and variable live load, as pointed out in the code Commentary. Again, we estimate the general eccentricity increase to be $0.01 \mathrm{~b}$.

(c) Torsional ground motion - The effect of torsional ground motion has been estimated by Newmark (3), and the main results of that paper have been reproduced by Newmark and Rosenblueth (2). First, a spectrum of torsional ground motion effect is estimated from a translational spectrum based roughly on the $1940 \mathrm{E} 1$ Centro N-S motion, with various assumptions about damping. The rotational component is obtained from the longitudinal derivative of the transverse shear wave. An equivalent eccentricity is then computed for a range of single-storey building types, all deforming in shear. Although the results apply to multistorey buildings, they are limited to buildings of twelve storeys or lower, because of the assumptions made about deformation.

The effective eccentricity depends on the aspect ratio of the building plan form (considering rectangular buildings), and very much on the translational period of the building. However, the results given in the references are computed for a value of $t_{b}$, the time taken for a shear wave to transverse the building, of 0.1 seconds. This evidently depends both on the size of the building and on the characteristics of the foundation material, but a more reasonable value would seem to be 0.01 seconds. As the torsional ground motion is directly proportional to $t_{b}$, the given results have to be reduced by 10. A further reduction might be justifiable, according to Newmark, because of an earlier assumption that waves of equal intensity cross the building both transversely and longitud- 
inally. Plotting Newmark's results for different building types and shapes leads to the general conclusion for symmetrical buildings that $e_{s}=0.25 b$ where the translational period $T>1.0$ seconds, and $e_{s}=0.05 \mathrm{~b}$ where $T<0.3$ seconds. Because of the uncertainties of the assumptions and the approximations in the analysis, a period-dependent result does not seem justifiable so that the constant value of $e_{s}=0.03 b$ has been chosen.

The various effects discussed in (a) and (c) above give eccentricity estimates which together sum to a value of $0.1 \mathrm{~b}$. In equation (1), this term has its greatest effect on buildings with only a small static eccentricity. As will be seen in the next section, the other terms in Eq. (1) could be unconservative for such buildings, so that $0.1 b$ looks after this as well as the accidental eccentricity effects.

\subsection{Coupling Between Torsional and Translational Modes}

The effect of coupling between torsional and translational modes where the natural frequencies are close has been well (7) (8). It can lead to severe torsional vibration. However (5) notes that for a tall building "The corners of a rectangular building can have a $95 \%$ increase in shear, as compared with 308 implied by a $5 \%$ eccentricity in the codes'.

Rosenblueth and Elorduy (4) derived curves showing the amplification of static eccentricity due to modal coupling. They are reprociuced in (2) and are shown in Fig. 2. They were obtained by analysing single storey buildings subjected to earthquakes having flat and hyperbolic acceleration spectra, though the choice of spectrum had little effect. These curves have been used as the basis of Eq. (1).

The curves in Fig. 2 were used to compute design eccentricities for a number of typical single storey building types. The results are plotted in Fig. 3, where it can be seen that most are bounded by the curves $n_{d}=1.7 n_{s}-n_{s}{ }^{2}$ and $n_{d}=n_{s}$. A bounding curve is also drawn in Fig. 3 representing the worst possible cases for a building square in plan (which is itself the worst of any rectangular shape). This curve is obtained from the peak values of Fig. 2, assuming the mass distribution is that for a square plate.

A further consideration helps in the choice of a code curve. For buildings with a fairly large eccentricity, rotational motion will shift the resultant of the inertia force away from the centre of mass. Consider the extreme situation of a rectangular building with a shear wall at one end only. Then the centre of rigidity will be at one end and the centre of mass will be at the centre so that $e_{s}=0.5 \mathrm{~b}$. Inertia forces will be greater further away from the centre of rigidity, as the motion is greater. For a rigid bar swinging about one end, the inertia force resultant moves to $b / \sqrt{3}$ from the centre of rigidity, or $e_{d}=0.577 \mathrm{~b}$. This limiting case lies just within the bounding parabola in Fig. 3 .
Clearly, more work needs to be done on the matter of amplification due to modal coupling. Dynamic analyses of typical multistorey buildings should be carried out to give further points for Fig. 3. And there is some preliminary indication that when large torsion occurs, the total lateral shear may be low (Ref. (2) indicates this, while (4) takes the opposite view). Nevertheless, based on the evidence available at the time of writing, Eqs. (1) and (2) are the most appropriate static design formulae.

\subsection{Combined Effects}

The torsion formulae of Eqs. (1) and (2) should be applied in only one direction at a time. It is not necessary to consider additional torsional effects due to earthquake motion at right angles to the direction under consideration.

\section{SHEAR INCREASE DUE TO TORSION}

Application of the torsion formulae will increase the transverse shear to be carried by some elements of a structure, particularly those elements farthest removed from the centre of rigidity. Some idea of the magnitude of this increase may be obtained from Fig. 4. The top curve represents the percentage increase in end-frame shear due to torsion, for symmetrical frame buildings of various aspect ratios $\mathrm{k}$ : the torsional shear is assumed to be distributed triangularly between the frames in both $N-S$ and $E-W$ directions. The limiting increase is $60 \%$ for long narrow buildings, reducing to $30 \%$ for square buildings.

As the frames are assumed to yield under the action of a severe earthquake, it would seem more reasonable to use a rectangular shear distribution among the frames rather than a triangular distribution, again taking the torsion in both directions. With this assumption, the end-frame torsional increase varies between $40 \%$ and $20 \%$, as shown by the second curve in Fig. 4.

The shear increase for a peripheral frame building is lower: the bottom curve in Fig. 4 shows the increase to vary between $20 \%$ and 10\%, depending on aspect ratio.

It should be remembered that when beam yield takes place, the end columns in a frame must carry high axial loads. In torsional situations, the corner columns of a building will therefore be subjected to severest attack.

For a symmetric building in which transverse forces are resisted by a shear wall at each end of the building, the increase in shear force due to torsion will be $20 \%$.

\section{CENTRES OF RIGIDITY}

The concept of a centre of rigidity is ill-defined and somewhat arbitrary. It has therefore been felt necessary to include clause 3.5 .3 which defined the centre of rigidity for a floor level in an irregular structure where the centre of rigidity is not obvious. Applying a couple at the top of a building has the same effect as 
applying the couple at the floor level under consideration, with the part of the building above that level removed. The centre of rigidity is then the centre of rotation at that floor under the action of the couple. The same point may also be defined (by the reciprocal theorem) as that point at which the application of a transverse force will cause no rotation of the floor level under consideration. This may sometimes be an easier approach to the calculation of the centre of rigidity.

\section{CONCLUSION}

The code static provisions for torsion may be used with confidence for reasonably regular buildings. They lead to an end frame shear force increase which may be as high as $40 \%$ but will more generally be of the order of $20 \%$. More investigation needs to be done on torsional effects, the most important areas being amplification due to modal coupling, the effect of torsional or differential ground motion, and the variation in position of the centre of rigidity in practice.

\section{REFERENCES}

1. 'Earthquake Resistant Regulations of the World - A World List 1973', International Association of Earthquake Engineering, Tokyo, 1973.

2. Newmark, N. M. and Rosenblueth, E., 'Fundamentals of Earthquake Engineering', Prentice-Hall, 1971.

3. Newmark, N.M., 'Torsion in Symmetric Buildings', Proc. 4th World Conf. on Earthquake Engineering, Chile, V.2., A-3 pp 19-32.

4. Elorduy, J. and Rosenblueth, E., - Torsiones Sismicas en Edeficios de Un Pisco', Report 164, Instituto de Ingenieria, Universidad Nacional Autonoma de Mexico, April 1968.

5. Hoerner, J. B., 'Modal Coupling and Earthquake Response of Tall Buildings', Calif. Inst. of Technology, Report EERL 71-07, 1971.

6. Penzien, J., 'Earthquake Response of Irregularly Shaped Buildings', Proc. 4 th World Conf. on Earthquake Engineering, Chile, V.2, A-3, pp 75-88.

7. Penzien, J. and Chopra, A. K., "Earthquake Response of Appendage on a MultiStorey Building", Proc. 3rd World Conf. on Earthquake Engineering, N.Z., V.II, pp 476-486, 1965 .

8. Skinner, R. I., Skilton, D.W.C. and Laws, D. A., 'Unbalanced Buildings, and Buildings with Light-Towers, Under Earthquake Forces', Proc. 3rd World Conference on Earthquake Engineering, N.Z. V.II, pp 586-602. 
$0.7 e_{s}-e_{s}^{2} / b \cdot 0.1 b+0.1 b=\frac{e_{d 1}}{d 2}$

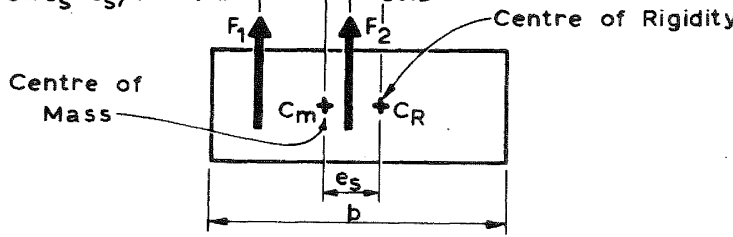

(a)

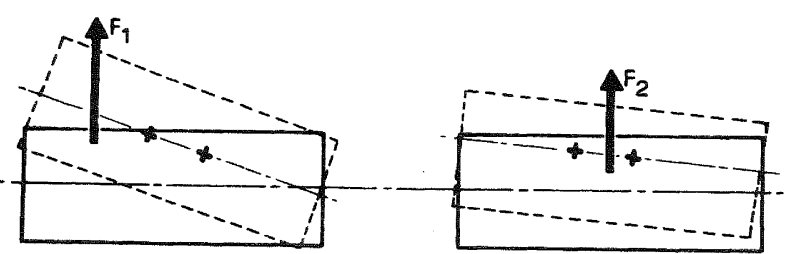

(b)

(c)

FIGURE 1: DEFINITION OF ECCENTRICITIES

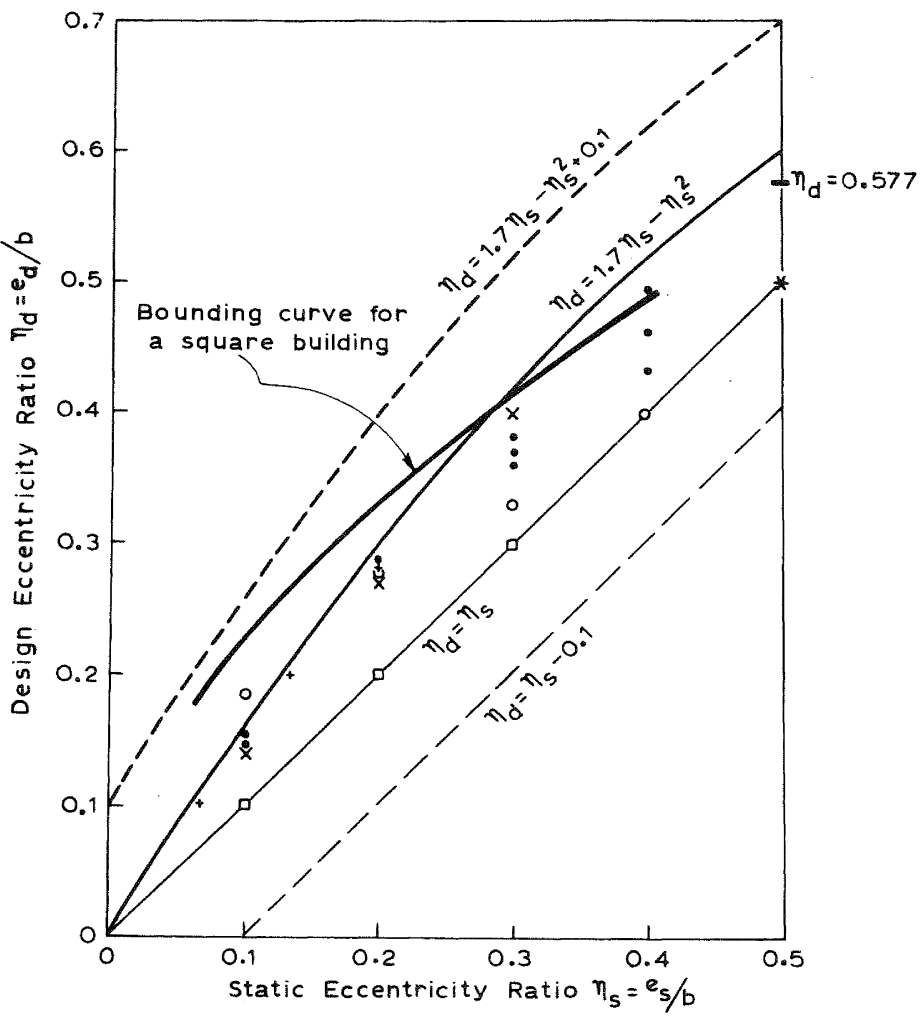

Shear Wall Building

Peripheral Frame

Frame Building

Eccentric Shear core

Frame with Wall at one end *

Four-Column Structures

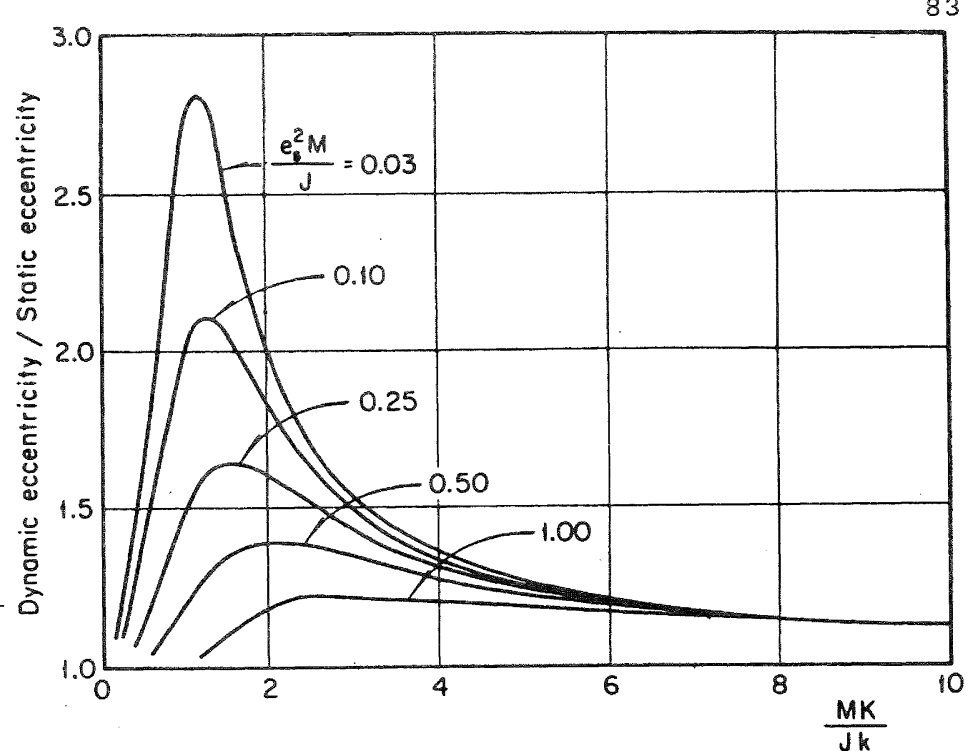

FIGURE 2: MAGNIFICATION FACTOR FOR ECCENTRICITY $M=$ mass

$J=$ moment of inertia

$K=$ torsional stiffness

$k=$ translational stiffness 